\title{
Scrotal and Orbital Involvement in Extramedullary De-differentiated Multiple Myeloma
}

\author{
Gavin Docherty, Barret Rush, Gary Victor, Greg Dueck
}

\begin{abstract}
About the Authors
Docherty G, PGY1 UBC Department of Ophthalmology, g.docherty@alumni.ubc.ca; Rush B, UBC Department of Medicine; Victor G, Kelowna General Hospital; and Dueck G, BCCA Regional Cancer Centre for the Southern Interior
\end{abstract}

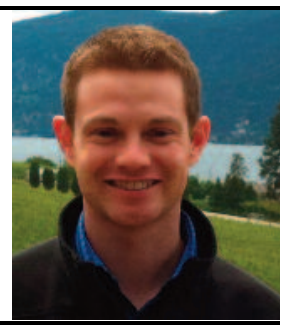

\section{Case Report}

A 71-year-old Indian male presented with a five-month history of progressive bilateral testicular swelling and swelling of the left orbit or one month duration. Nine months ago, he had undergone four cycles of chemotherapy (bortezomib, cyclophosphamide, and dexamethasone) for an immunoglobulin A (IgA) lambda multiple myeloma (3.8 grams per decilitre $[\mathrm{g} / \mathrm{dL}])$. This was stage 3 according to the International Staging System (ISS) and had no adverse cytogenetics. The patient had undergone six more cycles of treatment prior to presentation. Physical examination revealed gross distension of the left orbital tissues with violaceous colouring (Figure 1A). The abdomen was distended with

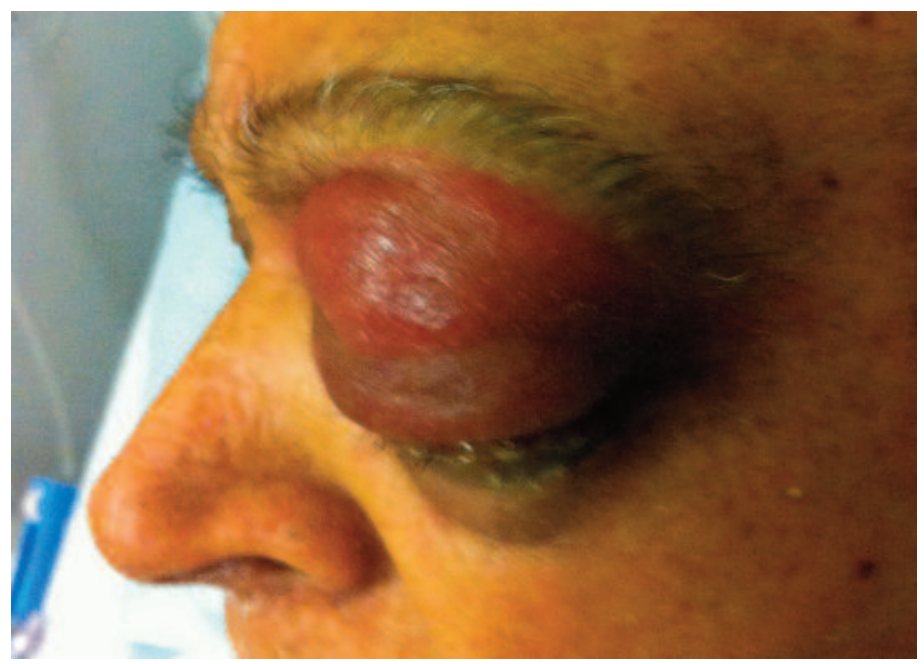

Figure 1A. Left orbital involvement in extramedullary de-differentiated myeloma. ascites. Genitourinary examination revealed testicular enlargement (Figure 1B). There was no erythema or warmth associated with either area. Laboratory tests failed to demonstrate monoclonal paraproteinemia, but his lactate dehydrogenase (LDH) level was elevated at 2068 units per litre (units/L). Abdominal paracentesis revealed a fluid cell count of 16,180 106/L and a fluid LDH of 10,591 units/L. Cytology showed CD138 positivity, $80 \%$ for Ki-67, and lambda light chain restriction. These findings supported a diagnosis of dedifferentiated extramedullary multiple myeloma. The prognosis and available management plans were discussed with the patient, and he was discharged to the community to be placed in the care of the palliative care team per his wishes.

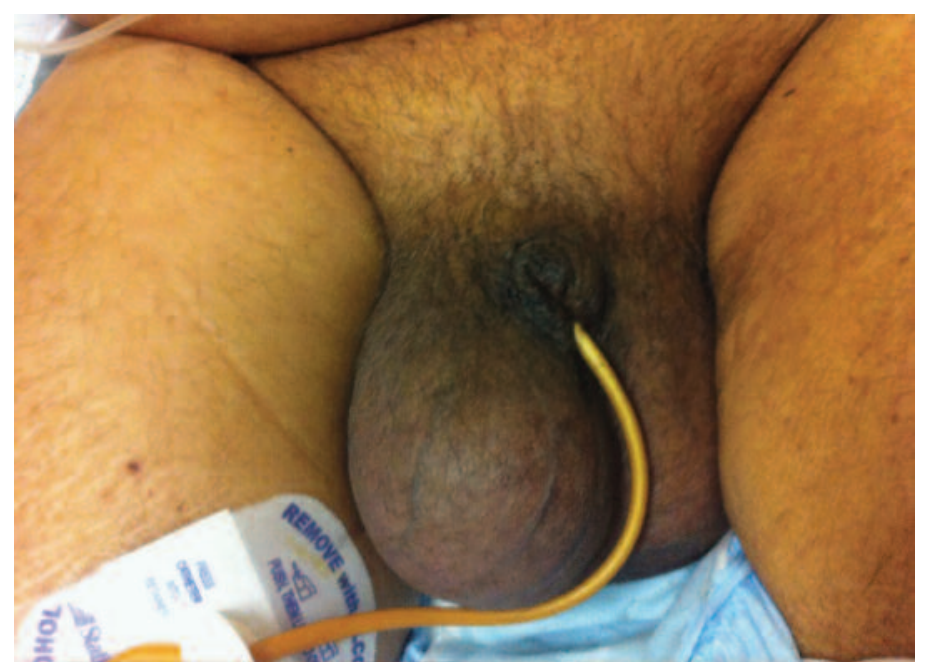

Figure 1B. Testicular involvement in extramedullary de-differentiated myeloma. 


\section{Discussion}

Multiple myeloma is usually characterized by production of a monoclonal protein by neoplastic plasma cells. This case report describes a patient who was diagnosed with multiple myeloma and demonstrated an unconfirmed complete response to therapy. His disease then progressed in an atypical manner, with extraosseous involvement of the scrotum, abdomen, and left orbit and lack of progressive monoclonal protein. This case represents de-differentiation of the patient's multiple myeloma, with subsequent escape from bone marrow and direct invasion of multiple tissues. The case was notable for loss of monoclonal protein in the serum, multiple extraosseous sites of involvement, and elevated LDH. Extramedullary tumour formation in multiple myeloma is a rare occurrence and rarely involves more than a single site. ${ }^{1}$ Varettoni et al. found that approximately $1 \%$ of patients presented with soft tissue or visceral disease. ${ }^{3} \mathrm{He}$ also suggested that the incidence of extramedullary myeloma may be increasing. ${ }^{3}$ Associated features of de-differentiated myeloma include high-grade histology, loss of CD56 expression, resistance to therapeutic regimens, and association with a poor prognosis. ${ }^{3}$ Dedifferentiation and extramedullary involvement in multiple myeloma appears to be becoming more common. ${ }^{4,5}$ This may be caused by alteration of adhesion molecules, which allows plasmablasts to escape from bone marrow. Dawson et al. proposed that novel biological therapies may alter the microenvironment and introduce an evolutionary pressure that predisposes to the clonal formation of cells that have acquired stromal independence and lost the ability to secrete intact immunoglobulin. ${ }^{4}$ Additionally, Yaccoby suggested that a subpopulation of malignant cells may survive chemotherapy because of their proximity to osteoclasts. ${ }^{5}$ These cells may then emerge as aggressive clones at a later date. Their ability to do so is attributed to the plasticity inherent in multiple myeloma cells, which allows them to de-differentiate into an immature and resilient phenotype. Furthermore, it is important to consider the possibility that atypical presentations during relapse may not be to the result of novel therapies but may represent the natural history of multiple myeloma in an era when patients are surviving longer as a result of current treatments.

Given the increasing incidence of de-differentiation and extramedullary involvement in multiple myeloma, it is important for clinicians to be aware of this mode of relapse.

\section{Acknowledgments}

We would like to thank the Pathology Department at the Kelowna General Hospital for their kind assistance, in particular Dr. D. Lesack and Mr. B. Stinson.

\section{References}

1. Jowitt SN, Jacobs A, Batman PA, et al. Atypical progression of multiple myeloma with extensive extramedullary disease. J Clin Pathol 1994;47:26971.

2. Cerny J, Fadare O, Hutchinson L, et al. Clinicopatholigical features of extramedullary recurrence/relapse of multiple myeloma. Eur J Haematol 2008;81(1): 65-9.

3. Varettoni M, Corso A, Pica G, et al. Incidence presenting features and outcome of extramedullary disease in multiple myeloma: a longitudinal study on 1003 consecutive patients. Ann Oncol 2010;21(2): 325-30.

4. Dawson, MA, Patil S, Spencer A. Extramedullary relapse of multiple myeloma associated with a shift in secretion from intact immunoglobulin to light chains. Haematologica 2007;92:143-4.

5. Yaccoby S. The phenotypic plasticity of myeloma plasma cells as expressed by dedifferentiation into an immature, resilient and apoptosis resistant phenotype. Clin Cancer Res 2005;11(21):7599-606. 